\title{
Functional Magnetic Resonance Study of Non-conventional Morphological Brains: malnourished rats
}

\author{
R. Martin ${ }^{1}$, F. Vazquez ${ }^{2}$, S.E. Solis-Najera ${ }^{2}$, O. Marrufo ${ }^{3}$, R. Godinez ${ }^{1}$, A. O. Rodriguez ${ }^{1}$ \\ ${ }^{1}$ Department of Electrical Engineering, Universidad Autonoma Metropolitana Iztapalapa, Av. San Rafael Atlixco 186, \\ Mexico, DF, 09340, Mexico,marsalrod@gmail.com,r.martin@ciencias.unam.mx \\ ${ }^{2}$ Faculty of Science, Universidad Nacional Autonoma de Mexico, Av. Universidad 3000, Circuito Exterior S/N, \\ Ciudad Universitaria, DF, 04510, Mexico. \\ ${ }^{3}$ Department of Neuro-Image, Instituto Nacional de Neurologia y Neurocirugia Manuel Velasco Suarez, \\ Insurgentes Sur 3877, Mexico, DF, 14269, Mexico.
}

\begin{abstract}
Malnutrition during brain development can cause serious problems that can be irreversible. Dysfunctional patterns of brain activity can be detected with functional MRI. We used BOLD functional Magnetic Resonance Imaging (fMRI) to investigate region differences of brain activity between control and malnourished rats. The food-competition method was applied to a rat model to induce malnutrition during lactation. A 7 T magnet was used to detect changes of the BOLD signal associated with changes in brain activity caused by the trigeminal nerve stimulation in malnourished and control rats. Major neuronal activation was observed in malnourished rats in several brain regions, including cerebellum, somatosensory cortex, hippocampus, and hypothalamus. Statistical analysis of the BOLD signals from various brain areas revealed significant differences in somatosensory cortex between the control and experimental groups, as well as a significant difference between the cerebellum and other structures in the experimental group. This study, particularly in malnourished rats, demonstrates increased BOLD activation in the cerebellum.
\end{abstract}

Keywords: Malnutrition, magnetic resonance, fMRI, brain activation, birdcage coil, trigeminal nerve.

\section{INTRODUCTION}

$\mathrm{T}$ HE INVESTIGATION of the process of cerebral maturation in mammals has provided the opportunity to investigate the mechanisms of cerebral development leading to brain damage, at both, morphological and neurotransmitter levels [1].

Studies of malnourished animal models, using postmortem histological and imaging analyses, have revealed a range of morphological changes, including a decrease in the prenatal brain size [2], a decrease in the postnatal brain size [3], fewer hippocampal neurons [4], diminished granular volume of cortex [5], abnormalities in the dendritic spines of the cerebellar cortex [6], reduced myelin content [7], and reduced synthesis of myelin-associated proteins [8].

Additional consequences of malnourishment on brain function include alterations in neurotransmitter systems. Specifically, previous research has found a reduction in serotonin levels [9], [10], increased concentrations of GABA in the hippocampus [11], a reduction in cholinergic cell numbers [12], increased levels of acetylcholinesterase (AChE) in the hippocampus [13], and a decrease in dopamine segregation in the hypothalamus, hippocampus, and cerebral cortex, all areas related to memory and learning [14], [15].

Functional in vivo recordings are typically performed with electroencephalographic methods, using both conventional superficial and invasive schemes [1]. Unfortunately, electroencephalographic methods can generate noisy signals that lead to the misinterpretation of results. The quality of electroencephalographic signals depends on the type and number of electrodes employed, which can cause problems when the electrode placement is inconsistent. A major caveat to consider is the possible damage to brain tissue that occurs at the moment of electrode insertion. A principal aim of brain functional studies is to image the brain at work using non-invasive techniques; therefore, magnetic resonance imaging (MRI) is a powerful tool with many advantages over other imaging modalities. MRI is a nonionizing technique that can provide both anatomical and functional information. Image contrast depends on intrinsic tissue properties, making it an ideal method to study brain activity. In this work, we used functional magnetic resonance imaging (fMRI), which provides a method for mapping brain functional activity based on the bloodoxygen-level-dependent effect (BOLD). We employed the BOLD fMRI technique to indirectly study the pattern of activity in the whole brain in an animal model of malnutrition.

The food-competition method is an established paradigm for provoking malnutrition in an animal model [16]. This method involves inducing malnutrition during lactation through food competition. A large number of pups cannot be sufficiently fed by one nursing mother, which causes a delay in the normal weight increase of the offspring.

Our principal aim was to study abnormal brain activity during a state of malnutrition. Therefore, we chose to stimulate the vibrissae-barrel axis both because of its suitability for studying structure, function, development and plasticity within the somatosensory cortex and for the functional and morphological correlation between the vibrissae and the barrels [17]. 


\section{MATERIALS \& METHODS}

\subsection{Animal preparation}

All animal procedures were performed according to the federal guidelines for Animal Care and were approved by the Ethical Committee of the Metropolitan Autonomous University, in accordance with international ethical standards. Twelve male Wistar rats (provided by the breeding-closed colony of Autonomous Metropolitan University Biological and Health Sciences Division, Mexico) were maintained on a 12:12 light cycle of controlled light-darkness at $22-25^{\circ} \mathrm{C}$ and 45 relative humidity.

Control and experimental groups consisted of 6 rats each (aged 18 to 21 days). For the malnutrition protocol, nursing rats fed twice as many pups as usual. Control rats weighed $41.94 \pm 4.9 \mathrm{~g}$, and malnourished rats weighted $29.09 \pm 3.3 \mathrm{~g}$. Pup weights in the experimental group correspond to a second-degree malnourished level based on the percentage of weight lost [1].

For fMRI experiments, rats were anesthetized in an acrylic box where isoflurane was administered at $2 \% \mathrm{l} / \mathrm{min}$ in $\mathrm{O}_{2}$, after which the rat's head was secured in a stereotaxic frame. Anesthesia was regulated between $1-1.5 \% 1 / \mathrm{min}$ in $\mathrm{O}_{2}$ during fMRI experiments. The effects of isoflurane have been evaluated using cerebral blood volume (CBV) and cerebral blood flow (CBF) [18-20]. Animal temperature variation was within $\pm 1{ }^{\circ} \mathrm{C}$; thus, the BOLD responses are not affected by changes in body temperature [21]. To perform MRI experiments, animals were placed in a custombuilt magnetic resonance (MR)-compatible Perspex rat cradle and secured into position using a bite bar and ear supports. Fig.1. shows the experimental setup.

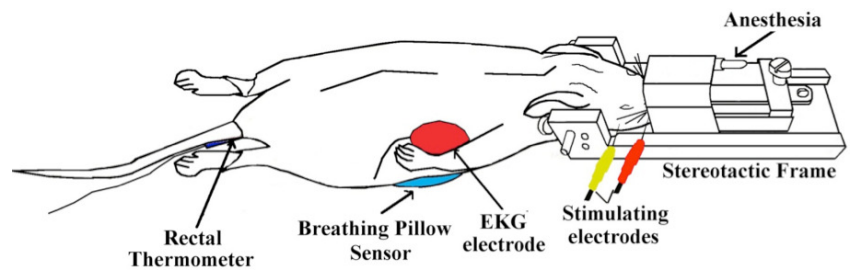

Fig.1. Animal setup employed for all BOLD experiments. Stimulating electrodes were placed on the whiskers (anode) and in the masticatory muscles (cathode). The anesthesia was delivered through the tube attached to the stereotactic frame. To minimize motion artifacts, the rat's head and ears were fixed to a stereotactic frame. This setup was also used to administer anesthesia at $1 \%$ of isoflurane with $2 \mathrm{l} / \mathrm{min}$ of $\mathrm{O}_{2}$ during all the fMRI experiment. EKG, breathing monitoring, and rat's temperature control $\left(37^{\circ} \mathrm{C} \pm 1{ }^{\circ} \mathrm{C}\right)$ were done with a small animal monitoring and gating system (Model 1025, SA Instruments, NY, USA).

\subsection{Trigeminal nerve stimulation}

The trigeminal nerve (the fifth cranial nerve, or CNV or CN5) contains both sensory and motor fibers and is responsible for sensation of the face and for motor functions such as biting, chewing, and swallowing. Sensory information from the face and body is processed by parallel pathways in the central nervous system. The whisker sensory system has been used as a model for studying this nerve in animal models, including rodents. This structure has aided the study of structure, function, development, and plasticity within the somatosensory cortex [17].

\subsection{MRI Volume Coil}

The design was optimized for a rat's head imaging experiments. The dimensions of the coil should fit the anatomical regions since the amplitude of the signal decays with distance. A conventional low pass birdcage coil was built and employed as a transceiver device. It consists of 16rung coil ( $10.5 \mathrm{~cm}$ long and a $5 \mathrm{~cm}$ diameter) to generate an optimal field uniformity. Rungs were made out of copper strips and distributed equidistantly around an acrylic cylinder and joined by two end circular rings. Fixed-value chip capacitors (American Technical Ceramics, series ATC $100 \mathrm{~B}$ nonmagnetic) of $18 \mathrm{pF}$ were soldered into the two conducting end rings for the generation of resonant modes. The volume coil was quadrature driven, so channels were matched to $50 \mathrm{Ohms}$ impedance of the radio frequency module. Two nonmagnetic trimmers (Voltronics, Corp: 1$33 \mathrm{pF}$, NMAJ30 0736) were used for this purpose: one trimmer was attached to each channel. Cable traps were built to improve the tuning and matching of the coil prototype. The cable trap was built by wrapping a section of coaxial cable into a tight helical shape with 8 turns and a $1.5 \mathrm{~cm}$ inner diameter, then exposing a small region of the outer conductor at each end of the helix and attaching a variable capacitor and tuning the resulting resonance to the Larmor frequency of our system, $300 \mathrm{MHz}$. Return loss plots for both channels were $300.45 / 300.05 \mathrm{MHz}$, measured with a network analyzer (Model 4396A, Hewlett Packard, Agilent Technologies, CA), as the loss return (S11) shown in Fig. 2 .

The experimental quality factor (Q) of the coil was determined by measuring the resonant frequency of the coil divided by the $3 \mathrm{~dB}$ bandwidth, $\Delta \omega$, with quarterwavelength coaxial cable at the input of the coil. The loaded $Q$ value was measured while the coil was loaded with a saline-filled spherical phantom ( $2 \mathrm{~cm}$ diameter). The $\mathrm{Q}$ with no load was around 59 and 35 when loaded, showing that the good performance was well maintained.

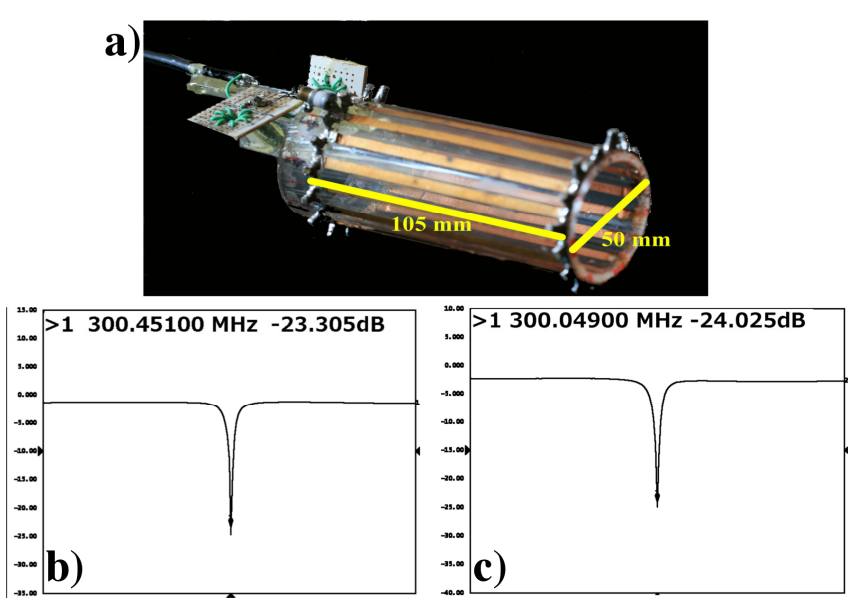

Fig.2. Photo of the 16 rung quadrature birdcage coil showing dimensions a), and the return loss obtained experimentally for each channel. 


\section{4. fMRI acquisition and data processing}

All experiments were performed on a $7 \mathrm{~T} / 21 \mathrm{~cm}$ Agilent system (Agilent Technologies, Inc., Palo Alto, CA) equipped with DirectDrive technology. We conducted anatomical and BOLD imaging experiments in the axial plane, as shown in Fig.3. Location images using a Fast Gradient Echo sequence with single-slice high contrast were obtained at the isocenter of the B0 to ensure that the imaging region was centered approximately at bregma $(-3.60 \mathrm{~mm})$ and to allow consistent imaging of the cortical and hippocampal regions. Anatomical images were acquired using a Spin Echo sequence with the following parameters: $\mathrm{TR} / \mathrm{TE}=3000 / 25 \mathrm{~ms}, \mathrm{FOV}=35 \times 35 \mathrm{~mm}$, matrix size $=$ $128 \times 128$, thickness $=0.3 \mathrm{~mm}$, gap $=0.2 \mathrm{~mm}$, slices $=20$, and $\mathrm{NEX}=4$. Rat brain images for BOLD analysis were obtained using a Multi-Echo Gradient Echo sequence and the following parameters: TR/TE $=107.82 / 4 \mathrm{~ms}, 15$ echoes (7.18ms between each echo), Flip angle $=20^{\circ}$, FOV $=$ $30 \times 30 \mathrm{~mm}$, matrix size $=128 \times 128$, thickness $=0.4 \mathrm{~mm}$, number of slices $=15$, and $\mathrm{NEX}=1$; thus, the spatial resolution is $0.23 \times 0.23 \times 0.4 \mathrm{~mm}^{3}$. Four volumes of images were acquired consecutively during excitation for a total time of acquisition around $60 \mathrm{~s}$.

BOLD fMRI experiments included both control and experimental groups. The left trigeminal nerve was stimulated using percutaneously inserted stainless steel electrodes; the cathode was positioned into the whiskers, and the anode was inserted into the masticatory muscles. The vibrissae barrel correlates to the trigeminal nerve, and this model gives excellent morphological and functional correlation between the whiskers and somatosensory cortex. A stimulator (Grass S-48 Stimulator, Grass Technologies, RI, USA) applied a train of current pulses $(500 \mathrm{mV}$ and $2 \mathrm{~mA}$ ) alternated between $60 \mathrm{~s}$ OFF and $60 \mathrm{~s}$ ON periods. These parameters have shown excellent results in the somatosensory cortex, as reported in [17].

To investigate the regions of interest in the somatosensory cortex, where neuronal activity is expected to change in response to sensory stimulation, all brain images were digitally processed using the toolbox SPM Mouse [24]. Data were overlaid onto anatomical images acquired in the same image plane; there was no possibility to use SPM template due the morphological changes in the brain of malnourished rats. To compare across control and experimental somatosensory cortices, we used a significance level of $\mathrm{p}<0.005$; for comparisons within the experimental group, a significance level of $p<0.05$ was used for estimating the SPM results. We took images in the coronal plane to produce the best representation and for easier localization of brain areas.

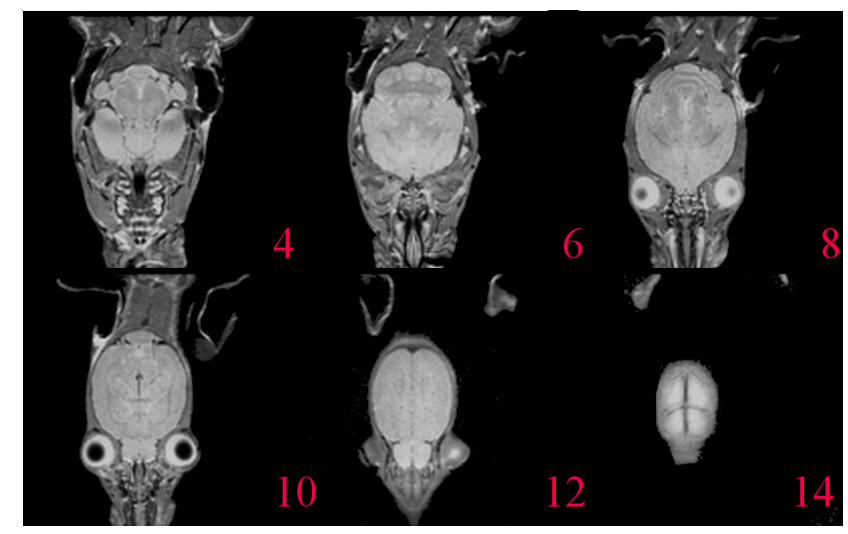

Fig.3. Example of original images taken in the axial plane for anatomical and BOLD analysis.

\section{RESULTS}

\section{1. fMRI maps}

To identify the areas in which neural activity might be altered, representative activation maps of stimuluscorrelated intensity changes were obtained from the brains of both groups. Fig.4. shows four different brain regions ranging from the posterior olfactory bulb to the cerebellum in both groups, highlighting regional differences in the activation across brain areas. Schematic diagrams from the Paxinos rat brain atlas were added to facilitate localization of the coronal sections within the rat brain [25]. Overall, the BOLD responses are temporarily distributed in a non-similar way in both groups; an important activation in the somatosensory cortex is present in the control group, as shown in Fig.3. In addition to the somatosensory cortex, brains of malnourished rats show activation of the hippocampus, hypothalamus, and cerebellum. In general, BOLD fMRI maps of malnourished rats show more activation across the entire brain than those of the control group.

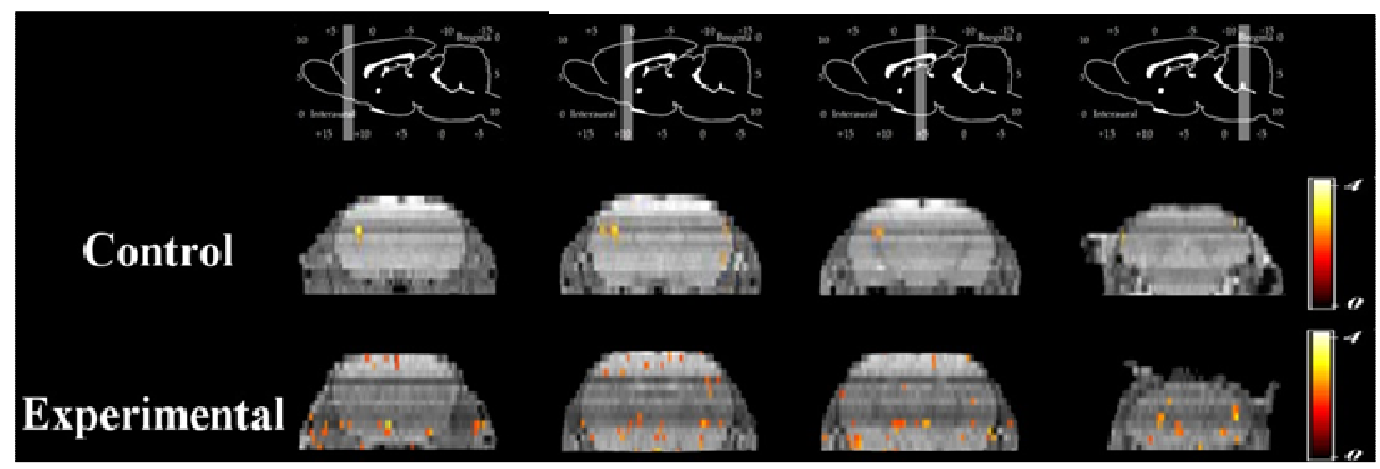

Fig.4. Coronal brain activation maps of the BOLD signal intensity of one rat from each group, showing activation. From left to right, we see areas ranging from the posterior region of the olfactory bulb to the cerebellum. The experimental group shows a random pattern of activation in comparison with the control group (expected response). 

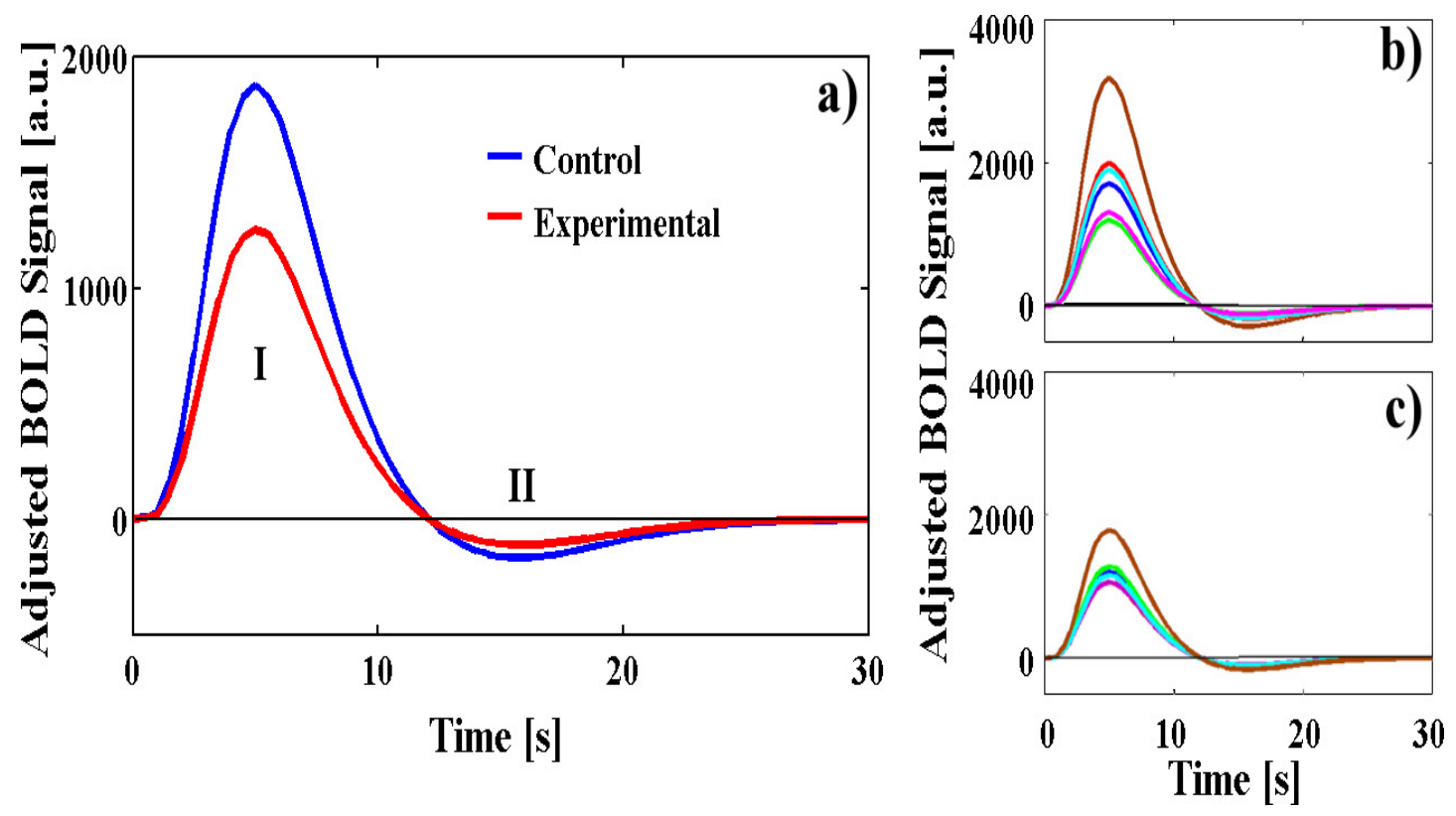

Fig.5. a) Adjusted mean BOLD signals from the somatosensory cortex obtained from b) the control group and d) the experimental group. Each group consisted of 6 rats each. Panel a) shows how the mean response was divided into two sections, the positive (I), from which the computed area was obtained, and the negative (II).

\subsection{BOLD response}

The BOLD response experiment uses a similar time delay for both groups and peaks approximately 5 seconds into the stimulation, Fig.5. Results for the experimental group suggest a greater level of oxygen consumption than in the control group. Fig.5.c) shows the averaged BOLD signal intensity time course for the control and experimental groups, which each included 6 rats (half of the total litter). For both groups, the signal sign changes after approximately $12 \mathrm{~s}$ when moving from region I to region II of Fig.5.c). The negative BOLD response caused by inhibition mechanisms was previously reported in [29]. A Student's t test was used to investigate the statistical independence of the measurements of the response amplitude. At the 0.05 level, the population means $\left(\right.$ mean $_{\text {control }}=1879.39$, and mean $n_{\exp }=$ 1254.34) are significantly different. To quantify the BOLD response of the malnourished rats, we used the method reported in [30]. The response amplitudes shown in Fig.5.c) were calculated from the peak of the BOLD response intensity for each mean group response. Additionally, the response integral was calculated as the product of the amplitude and the full width at half maximum (FWHM), which was determined to be 5638.18 and 3763.01 for the control and malnourished rats, respectively. A simple trapezoid-integration under the positive curve was also computed; the results were 20460.15 and 13708.85 for control and malnourished rats, respectively. The area under the curves indicates a clear increase in the neuronal activity for the malnourished rats over the control group.

BOLD responses within the experimental group were also analyzed. Fig.6. shows BOLD responses for several brain structures: the cerebellum, hypothalamus, hippocampus, and somatosensory cortex. We calculated the same measurements of area under the positive curve of the mean responses, and the results obtained by simple trapezoid-rule integration were as follows: cerebellum $\approx 21625.49$, hypothalamus $\approx 15606.45$, hippocampus $\approx 15272.86$, and somatosensory cortex $\approx 13708.85$. A similar pattern was obtained with the FWHM method; for the malnourished group, the results were cerebellum $\approx 5936.09$, hypothalamus $\approx 4283.90$, hippocampus $\approx 4192.33$, and somatosensory cortex $\approx 3763.01$

Individual and averaged BOLD time course signals were obtained for each subject in different brain areas (Fig.5. and Fig.6.) as obtained by Zumer et al. [31]. This signal is the typical (canonical) BOLD impulse response, and it is considered as the sum of the accumulated responses in the regions of interest [32]. Maximum peaks of these signals were obtained (in the averaged signals) to improve our analysis. In almost all signals, there are no significant differences observed until the maximum intensity of signal has been reached (Fig.7.).

An ANOVA was also performed to these data, using a significance level of $p<0.05$, and the results are summarized in Table 1. We found a significant difference only between the cerebellum response and the other three structures; no statistically significant differences were identified for the other regions. The hypothalamus and hippocampus show very similar values. Our findings are consistent with previous results obtained using invasive techniques that identified the cerebellum as one of the structures more strongly affected by malnutrition [5].

Malnutrition also affects structures involved in learning and memory processes, including the hippocampus and hypothalamus [2-14]. 
Anatomical images for both groups reveal a marked difference in the third ventricle and cerebellum, as shown in Fig.8. We used these images, because the third ventricle was more visible in those slices with help of an expert and according to Paxino's rat brain atlas we approach to the same plane, so an image processing script based on growing region was performed to analyze the number of pixels contained in the region of interest only in $2 \mathrm{D}$, we obtained that in the third ventricle, this is, we took the darker pixels contained in the region; the mean number of pixels was $109.33 \pm 15.76$ and $209.33 \pm 40.34$ pixels for the control group and the experimental group, respectively which represents a difference of almost $90 \%$ of number of pixels Additionally, in that same plane and in $2 \mathrm{D}$, the analysis for the cerebellum, the approximate number of pixels was $2017.33 \pm 28.87$ and $1994.67 \pm 58.24$ for the control group and the experimental group, respectively; this is a difference of approximately $3 \%$. These results corroborate the model that a malnourished brain has greater "water" areas (in our case for the third ventricle) and displays signs of abnormal brain development (especially in the cerebellum) [34]. Therefore, a three-dimensional rendering process was computed to observe anatomical differences between representative subjects of the groups.
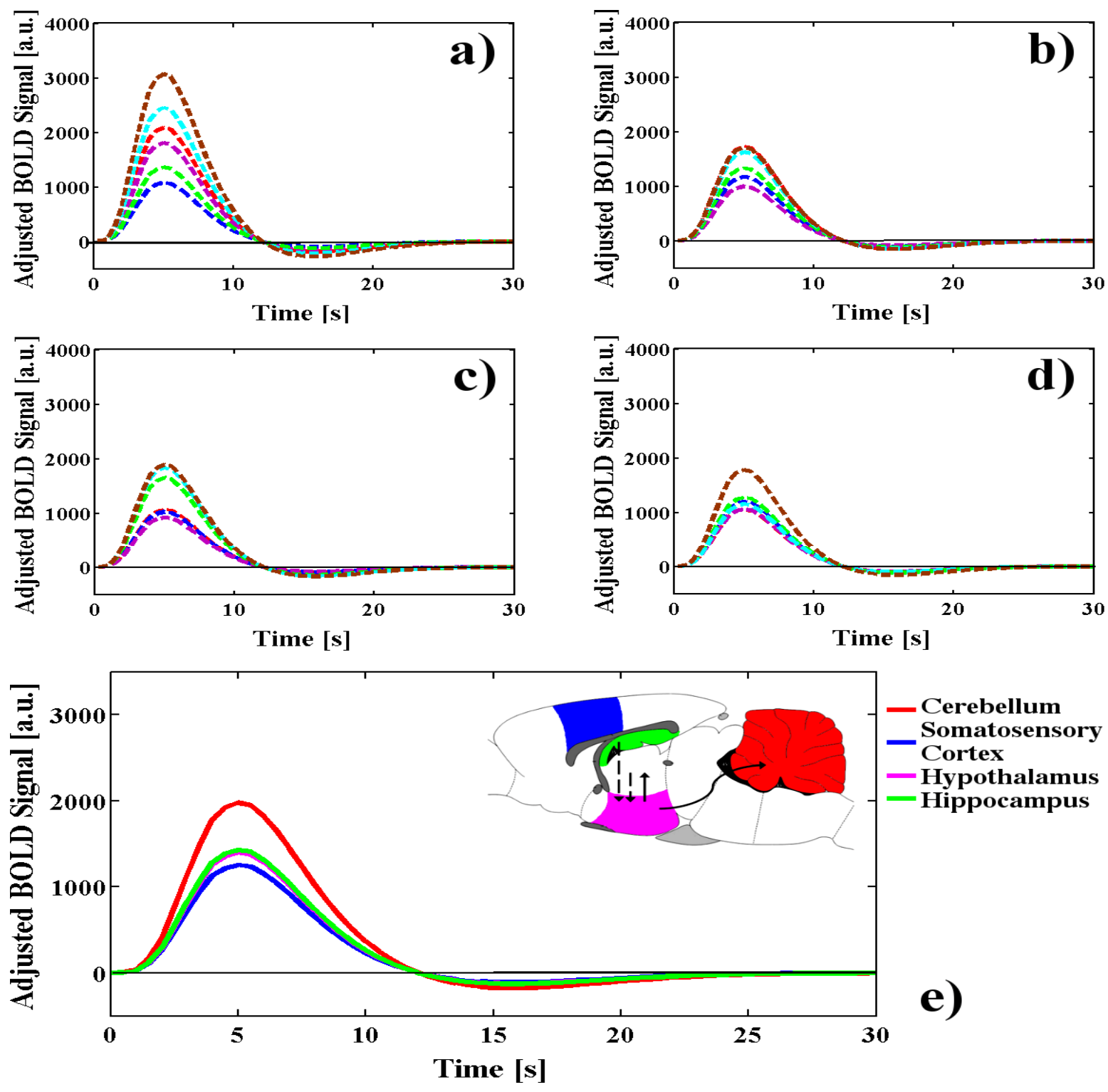

Fig.6. Adjusted BOLD signals for several brain areas from the experimental (malnourished) group: a) cerebellum, b) hypothalamus, c) hippocampus, and d) somatosensory cortex. Additionally, the mean BOLD signal from each brain area is presented in e). All figures use the same maximum intensity scale to fully highlight differences between structures. The lower panel shows a brain diagram depicting the areas from which the signals were taken and also indicates the afferent (dashed arrow) and the efferent pathways (solid arrows) to and from the hippocampus. 


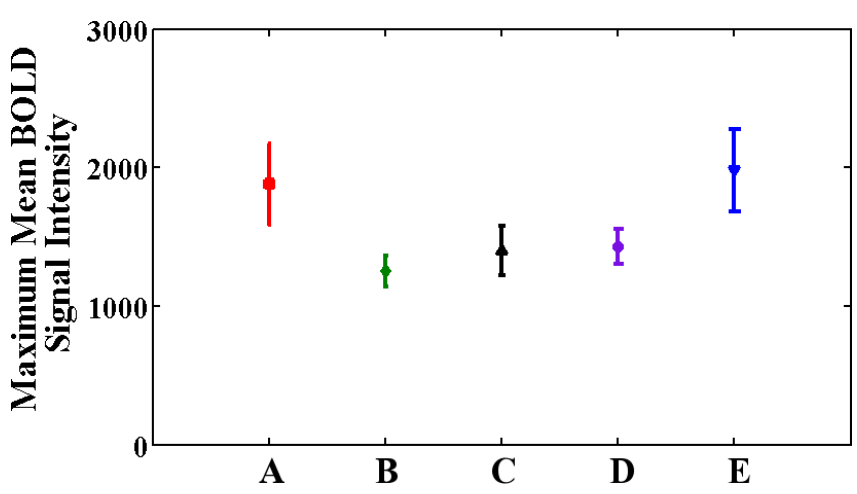

Fig.7. Comparison of the maximum signal intensities obtained from the mean BOLD responses with their respective standard deviation, in the different regions of interest; A - Somatosensory Cortex of control group, while B - Somatosensory Cortex, C Hippocampus, D - Hypothalamus, and E - Cerebellum from the experimental group.

Table 1. ANOVA analysis results for signals from the four different brain areas. The sig value indicates the significant difference between areas $(1=$ significant difference, $0=$ no significant difference).

\begin{tabular}{llll}
\hline & $\begin{array}{l}\text { Mean } \\
\text { Difference }\end{array}$ & Prob. & Sig \\
$\begin{array}{l}\text { Cerebellum - } \\
\text { Somatosensory } \\
\text { Cortex }\end{array}$ & 724.359 & 0.0067 & 1 \\
$\begin{array}{l}\text { Hippocampus - } \\
\text { Somatosensory } \\
\text { Cortex }\end{array}$ & 143.104 & 0.0952 & 0 \\
$\begin{array}{l}\text { Hippocampus - } \\
\text { Cerebellum }\end{array}$ & 581.255 & 0.0178 & 1 \\
$\begin{array}{l}\text { Hypothalamus - } \\
\text { Somatosensory } \\
\text { Cortex }\end{array}$ & 173.627 & 0.0919 & 0 \\
$\begin{array}{l}\text { Hypothalamus - } \\
\text { Cerebellum }\end{array}$ & 550.732 & 0.0215 & 1 \\
$\begin{array}{l}\text { Hypothalamus }- \\
\text { Hippocampus }\end{array}$ & 30.523 & 0.0999 & 0 \\
\hline
\end{tabular}

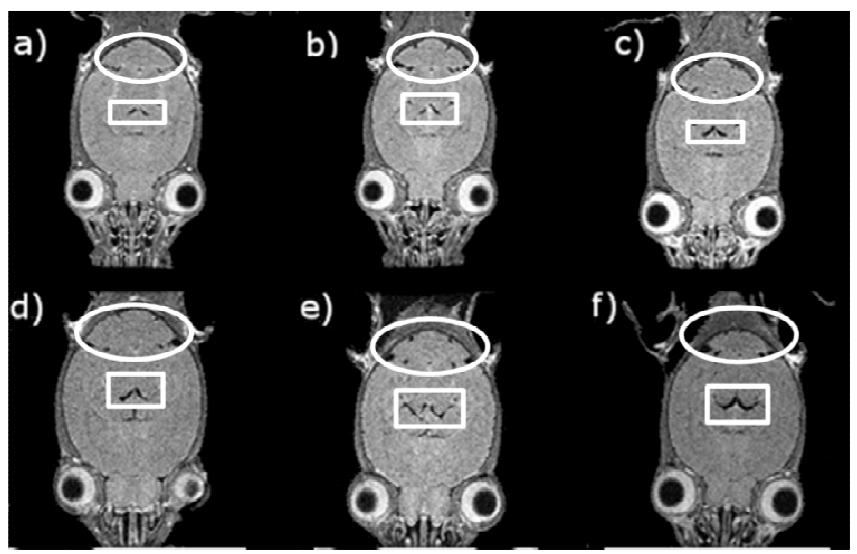

Fig.8. Anatomical images for the groups: a), b), and c) represent rats from the control group; d), e), and f) represent images from the experimental group. For the experimental group, three representative rats are shown, and the images indicate different degrees of abnormality in the cerebellum (circle) and the area around the third ventricle (box).

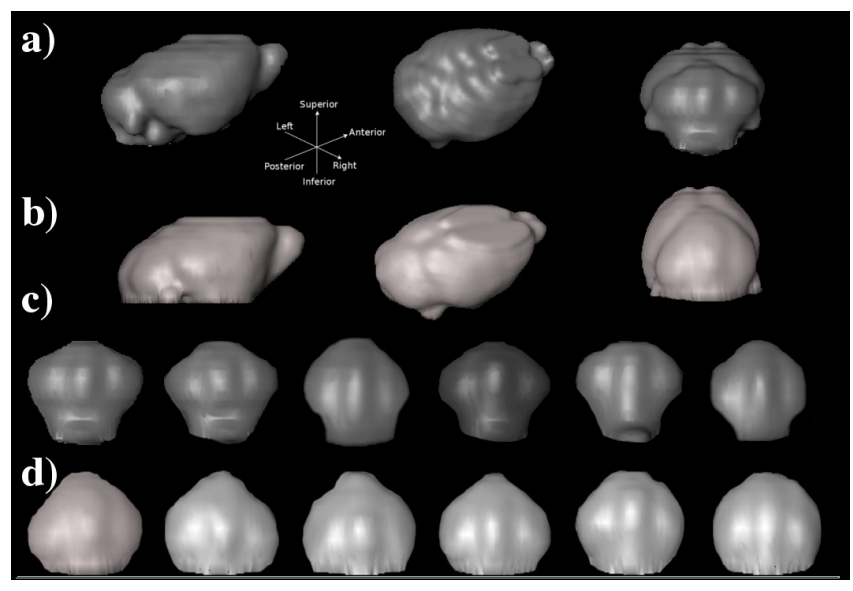

Fig.9. Three-dimensional reconstructions from the anatomical images obtained from one rat of the control a) and experimental b) groups. An important difference between the groups is visible at the cerebellum c) and d) where are shown all cerebellums of control and experimental groups, in which a notorious abnormal development can be noticed.

Fig.9. shows the 3D segmented model to enhance the differences in brain structures. Fig.9.a) shows a normal rat brain with regular growing that clearly depicts significant differences in brain structures, primarily at the level of cerebellum development. Fig.9.b) shows the control group and Figs.9.c) and 9.d) enhance anatomical abnormalities in the development of the cerebellum.

\section{DISCUSSION}

Images for functional analysis were obtained with a homebuilt dedicated quadrature volume coil for rats' size; there are limited references of fMRI with a transceiver volume coil. To our knowledge, this is the first fMRI study on malnutrition using an animal model. The results demonstrate similar temporal distribution of BOLD responses in malnourished and control rats. Negative BOLD responses for the two groups of rats can also be interpreted as being caused by inhibition mechanisms.

Our results indicate an abnormal increase in the electrical activity in several regions of the cerebrum. This effect is likely a result of a decrease in the neural inhibitory discharges of the inhibitory postsynaptic potentials (IPSPs) and/or an increase in the excitatory postsynaptic potentials (EPSPs) [26]. Results reported by Segura and collaborators [27] provided evidence for a demyelinating process in both the peripheral nervous system and at the encephalic level of malnourished rats. We suspect that malnutrition preferentially affects the inhibitory interneurons and that the demyelination process produces a decrease in the IPSPs that subsequently increases the neuronal activity in a random way, as depicted in Fig.4. These results cannot yet indicate whether the abnormal activation observed in different brain regions is a consequence of brain tissue damage or the result of an adaptive process of neural plasticity. The pronounced changes in activity suggest the existence of latent synaptic pathways that are not normally expressed but are unmasked in the brain of a malnourished subject.

The results were not as expected because we assumed that there would be limited alterations at the somatosensory cortex and did not expect to observe significant changes in 
the function of other structures, but still are congruent with previous studies, because alterations of hypothalamus and hippocampus are related to inhibition of hunger. The brain changes we observed may result from disruptions in the signaling mechanisms of neurotransmitters, such as GABA (inhibitory) or glutamate (excitatory). Additionally, other neurotransmitter pathways, including those of acetylcholine (ACh), dopamine, or noradrenaline, may be impaired.

Our results imply that impairments may be induced in the hypothalamic pathway and other structures, as shown in Fig.6.

This study provides an initial approach to study this health problem and the resulting consequences for brain function. At this stage, it is not possible to determine whether the changes in the brain correspond to brain damage or to a process of adaptive plasticity.

\section{CONCLUSION}

We used BOLD fMRI and trigeminal nerve stimulation to study the BOLD changes during sensory stimulation in malnourished rats. This study is one of the first performed in malnourished rats and demonstrates increased BOLD activation in the cerebellum. Further investigations using spectroscopy for the measurement of GABA and glutamate, or using DTI, are necessary to determine whether plasticity, demyelination, or the combination of the two is the main cause of the activation of the brain areas reported here. These results may lead to new treatments for this health problem or the development of other possible rehabilitation procedures.

\section{ACKNOWLEDGMENT}

R. M., F. V., and O. M. would like to thank CONACYT Mexico for $\mathrm{Ph}$. D. scholarships and grant number 112092.

\section{REFERENCES}

[1] Medina, M.T., Amador, C., Hernández-Toranzo, R., Hesse, H., Holden, K.R., Morales-Ortíz, A., Rodríguez-Salinas, L.C. (2008). Neurologic Consequences of Malnutrition. New York: Demos Medical Publishing.

[2] Zeman, F.J., Heng, H., Hoogenboom, E.R., Kavlock, R.J., Mahboob, S. (1986). Cell number and size in selected organs of fetuses of rats malnourished and exposed to nitrofen. Teratogenesis, Carcinogenesis, and Mutagenesis, 6 (4), 339-347.

[3] Fukuda, M.T.H., Françolin-Silva, A.L., Sousa Almeida, S. (2002). Early postnatal protein malnutrition affects learning and memory in the distal but not in the proximal cue version of the Morris water maze. Behavioural Brain Research, 133 (2), 271-277.

[4] Lister, J.P., Blatt, G.J., DeBassio, W.A., Kemper, T.L., Tonkiss, J., Galler, J.R., Rosene, D.L. (2005). Effect of prenatal protein malnutrition on numbers of neurons in the principal cell layers of the adult rat hippocampal formation. Hippocampus, 15 (3), 393-403.

[5] Hillman, D.E., Chen, S. (1981). Vulnerablity of cerebellar development in malnutrition-I. Quantation of layer volume and neuron numbers. Neuroscience, 6 (7), 1249-1262.
[6] Benitez-Bribiesca, L., De la Rosa-Alvarez, I. Mansilla-Olivares, A. (1999). Dendritic spine pathology in infants with severe protein-calorie malnutrition. Pediatrics, 104 (2), e21.

[7] Reddy, P.V., Das, A., Sastry, P.S. (1979). Quantitative and compositional changes in myelin of undernourished and protein malnourished rat brains. Brain Research, 161 (2), 227-235.

[8] Montanha-Rojas, E.A., Ferreira, A.A., Tenório, F., Barradas, P.C. (2005). Myelin basic protein accumulation is impaired in a model of protein deficiency during development. Nutritional Neuroscience, 8 (1), 49-56.

[9] Mazer, C., Muneyyirci, J., Taheny, K., Raio, N., Borella, A., Whitaker-Azmtia, P. (1997). Serotonin depletion during synaptogenesis leads to decreased synaptic density and learning deficits in the adult rat: A possible model of neurodevelopmental disorders with cognitive deficits. Brain Researc, 760 (1-2), 6873.

[10] Chen, J.C., Turiak, G., Galler, J., Volicer, L. (1997). Postnatal changes of brain monoamine levels in prenatally malnourished and control rats. International Journal of Developmental Neuroscience, 15 (2), 257263.

[11] Chang, Y.M., Galler, J.R., Luebke, J.I. (2003). Prenatal protein malnutrition results in increased frequency of miniature inhibitory postsynaptic currents in rat CA3 interneurons. Nutritional Neuroscience, 6 (4), 263-267.

[12] Nakagawasai, O. (2005). Behavioral and neurochemical alterations following thiamine deficiency in rodents: Relationship to functions and cholinergic neurons. Yakugaku Zasshi, 125 (7), 549 554.

[13] Cermak, J.M., Holler, T., Jackson, D.A., Blusztajn, J.K. (1998). Prenatal availability of choline modifies development of the hippocampal cholinergic system. FASEB Journal, 12 (3), 349-357.

[14] Zimmer, L., Delpal, S., Guilloteau, D., Aïoun, J., Durand, G., Chalon, S. (2000). Chronic n-3 polyunsaturated fatty acid deficiency alters dopamine vesicle density in the rat frontal cortex. Neuroscience Letters, 284 (1-2), 25-28.

[15] Chalon, S., Vancassel, S., Zimmer, L., Guilloteau, D., Durand, G. (2001). Polyunsaturated fatty acids and cerebral function: Focus on monoaminergic neurotransmission. Lipids, 36 (9), 937-944.

[16] Ortíz, R., Cortés, E., Pérez, L., González, C., Betancourt, M. (1996). Assessment of an experimental method to induce malnutrition by food competition during lactation. Medical Science Research, 24, 843846.

[17] Just, N. Petersen, C., Gruetter, R. (2010). BOLD responses to trigeminal nerve stimulation. Magnetic Resonance Imaging, 28 (8), 1143-1151.

[18] Wegener, S., Wong, E.C. (2008). Longitudinal MRI studies in the isoflurane-anesthetized rat: Long-term effects of a short hypoxic episode on regulation of cerebral blood flow as assessed by pulsed arterial spin labelling. NMR in Biomedicine, 21 (7), 696-703. 
[19] Sicard, K., Shen, Q., Brevard, M.E., Sullivan, R., Ferris, C.F., King, J.A., Duong, T.Q. (2003). Regional cerebral blood flow and BOLD responses in conscious and anesthetized rats under basal and hypercapnic conditions: Implications for functional MRI studies. Journal of Cerebral Blood \& Flow Metabolism, 23 (4), 472-481.

[20] Kim, T., Masamoto, K., Fukuda, M., Vazquez, A., Kim, S.G. (2010). Frequency-dependent neural activity, CBF, and BOLD fMRI to somatosensory stimuli in isoflurane-anesthetized rats. Neuroimage, 52 (1), 224-233.

[21] Vanhoutte, G., Verhoye, M., Van der Linden, A. (2006). Changing body temperature affects the T2* signal in the rat brain and reveals hypothalamic activity. Magnetic Resonance in Medicine, 55 (5), 1006-1012.

[22] Hyder, F., Behar, K.L., Martin, M.A., Blamire, A.M., Shulman, R.G. (1994). Dynamic magnetic resonance imaging of the rat brain during forepaw stimulation. Journal of Cerebral Blood \& Flow Metabolism, 14 (4), 649-655.

[23] Yang, X., Hyder, F., Shulman, R.G. (1996). Activation of single whisker barrel in rat brain localized by functional magnetic resonance imaging. Proceedings of the National Academy of Sciences USA, 93 (1), 475 478.

[24] Sawiak, S.J., Wood, N.I., Williams, G.B., Morton, A.J., Carpenter, T.A. (2009). SPMMouse: A new toolbox for SPM in the animal brain. In ISMRM 17th Scientific Meeting \& Exhibition, Honolulu, US, 18-24 April 2009. ISMRM, 6264.

[25] Paxinos, G., Watson, Ch. (1998). The Rat Brain in Stereotaxic Coordinates, 4th ed. Academic Press.

[26] Kandel, E.R. (2000). Principles of Neural Science. McGraw-Hill.

[27] Segura, B., Guadarrama, J.C., Pratz, G., Mercado, V., Merchant, H., Cintra, L., Jiménez, I. (2004). Conduction failure of action potentials in sensory sural nerves of undernourished rats. Neuroscience Letters, 354 (3), 181-184.

[28] Silva, A.C., Koretsky, A.P. (2002). Laminar specificity of functional MRI onset times during somatosensory stimulation in rat. Proceedings of the National Academy of Sciences USA, 99 (23), 1518215187.
[29] Vandervliet, E., Nagels, G., Heinecke, A., Van Hecke, W., Leemans, A., Sijbers, J., Parizel, P.M. (2006). On the cause and mechanisms of the negative BOLD response in fMRI. In ESMRMB 2006: 23rd Annual Scientific Meeting, Warsaw, Poland, 21-23 September 2006. ESMRMB, 624.

[30] Lindquist, M.A., Meng Loh, J.M., Atlas, L.Y., Wager, T.D. (2009). Modeling the hemodynamic response function in fMRI: Efficiency, bias and mis-modeling. Neuroimage, 45 (1 Suppl), S187-S198.

[31] Zumer, J.M., Brookes, M.J., Stevenson, C.M., Francis, S.T., Morris, P.G. (2010). Relating BOLD fMRI and neural oscillations through convolution and optimal linear weighting. Neuroimage, 49 (2), 1479-1489.

[32] Henson, R., Friston, K. (2007). Convolution models for fMRI. In Statistical Parametric Mapping: The Analysis of Functional Brain Images. Elsevier, 178192.

[33] Yeşilyurt B., Uğurbil K., Uludağ K. (2008). Dynamics and nonlinearities of the BOLD response at very short stimulus durations. Magnetic Resonance Imaging, 26 (7), 853-862.

[34] Gunston, G.D., Burkimsher, D., Malan, H., Sive, A.A. (1992). Reversible cerebral shrinkage in kwashiorkor: An MRI study. Archives of Disease in Childhood, 67 (8), 1030-1032.

[35] Birn, R.M., Saad, Z.S., Bandettini, P.A. (2001). Spatial heterogeneity of the nonlinear dynamics in the FMRI BOLD response. Neuroimage, 14 (4), 817-826.

[36] Sizonenko, S.V., Babiloni, C., de Bruin, E.A., Isaacs, E.B., Jönsson, L.S., Kennedy, D.O., Latulippe, M.E., Hohajen, M.H., Moreines, J., Pietrini, P., Walhovd, K.B., Winwood, R.J., Sijben, J.W. (2013). Brain imaging and human nutrition: Which measures to use in intervention studies? British Journal of Nutrition, 110 (1), S1-S30.

[37] Van Camp, N., Verhoye, M., Van der Linden, A. (2006). Stimulation of the rat somatosensory cortex at different frequencies and pulse widths. NMR in Biomedicine, 19 (1), 10-17.

[38] Bullmore, E., Sporns, O. (2009). Complex brain networks: Graph theoretical analysis of structural and functional systems. Nature Reviews Neuroscience, 10 (3), 186-198.

Received January 06, 2015. Accepted July 30, 2015. 